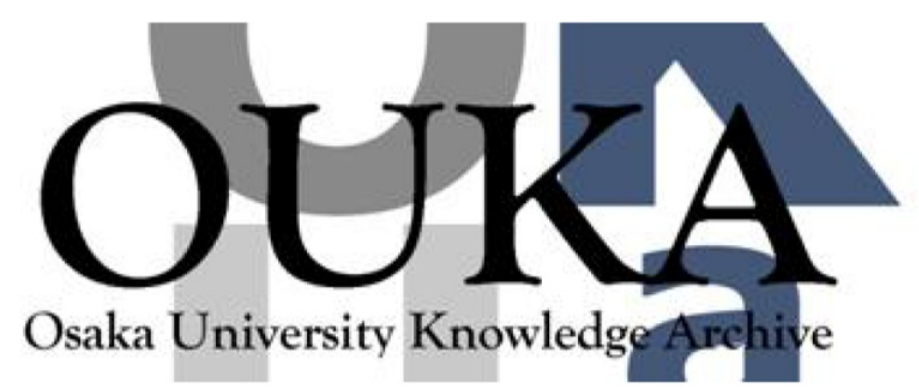

\begin{tabular}{|c|l|}
\hline Title & $\begin{array}{l}\text { Integrated implosion/heating studies for } \\
\text { advanced fast ignition }\end{array}$ \\
\hline Author(s) & $\begin{array}{l}\text { Norreys, P.A.; Lancaster, K. L. ; Murphy, C.D. et } \\
\text { al. }\end{array}$ \\
\hline Citation & Physics of Plasmas. 11(5) p. 2746-p. 2753 \\
\hline Issue Date & $2004-05$ \\
\hline oaire:version VoR \\
\hline URL & https://hdl. handle.net/11094/3089 \\
\hline rights & \\
\hline Note & \\
\hline
\end{tabular}

Osaka University Knowledge Archive : OUKA

https://ir. Library. osaka-u. ac. jp/

Osaka University 


\title{
Integrated implosion/heating studies for advanced fast ignition ${ }^{\text {a) }}$
}

\author{
P. A. Norreys, ${ }^{1, b)}$ K. L. Lancaster, ${ }^{1,2}$ C. D. Murphy, ${ }^{1,2}$ H. Habara, ${ }^{1}$ S. Karsch, ${ }^{1}$ \\ R. J. Clarke, ${ }^{1}$ J. Collier, ${ }^{1}$ R. Heathcote ${ }^{1}$ C. Hemandez-Gomez, ${ }^{1}$ S. Hawkes, ${ }^{1}$ D. Neely, ${ }^{1}$ \\ M. H. R. Hutchinson, ${ }^{1,2}$ R. G. Evans, ${ }^{2}$ M. Borghesi, ${ }^{3}$ L. Romagnani, ${ }^{3}$ M. Zepf, ${ }^{3}$

(Received 6 November 2003; accepted 23 January 2004; published online 23 April 2004)

\begin{abstract}
Integrated experiments to investigate the ultrafast heating of implosions using cone/shell geometries have been performed at the Rutherford Appleton Laboratory. The experiments used the $1054 \mathrm{~nm}$, nanosecond, $0.9 \mathrm{~kJ}$ output of the VULCAN Nd:glass laser to drive $486 \mu \mathrm{m}$ diameter, $6 \mu \mathrm{m}$ wall thickness $\mathrm{Cu}$-doped deuterated plastic (CD) shells in 6-beam cubic symmetry. Measurements of the opacity of the compressed plasma using two-dimensional spatially resolved Ti- $K_{\alpha}$ x-ray radiography suggest that densities of $4 \mathrm{~g} \mathrm{~cm}^{-3}$ and areal densities of $40 \mathrm{mg} \mathrm{cm}^{-2}$ were achieved at stagnation. Upper limits on the heating with both $1 \mathrm{ps}$ and $10 \mathrm{ps}$ pulses were deduced from the fluorescent yield from the $\mathrm{Cu}$ dopant. The data suggest that control of the preformed plasma scale-length inside the cone is necessary for efficient coupling to the compressed plasma. (C) 2004 American Institute of Physics. [DOI: 10.1063/1.1688790]
\end{abstract}

\section{INTRODUCTION}

One of the most attractive fast-track paths to fusion energy is now being explored in laser fusion research - the advanced fast ignition concept. ${ }^{1}$ The first demonstrations of this new concept have been reported at the Rutherford Appleton Laboratory (RAL) (Ref. 2) in the United Kingdom and at the Institute of Laser Engineering (ILE), Osaka University in Japan. $^{3}$ A $60 \mathrm{~J}, 100 \mathrm{TW}$ short-duration laser pulse was coupled to a novel target geometry that allowed simultaneous compression and significant heating. The results indicated a highly efficient coupling of the short pulse laser energy to the heating of the compressed plasma.

The next step was to conduct experiments at a laser power equivalent to a full-scale ignition system, albeit with a pulse duration and energy $1 / 20$ of that required for ignition. The first equivalent power experiments using a $350 \mathrm{~J}$, PetaWatt class laser system as the heating source showed a three orders of magnitude enhancement of fusion products. These results confirmed that the high heating efficiency was maintained as the short-pulse laser energy was substantially

\footnotetext{
a) Paper KI2 5, Bull. Am. Phys. Soc. 48, 171 (2003).

${ }^{\mathrm{b})}$ Invited speaker.
}

increased. ${ }^{4}$ Measurements using perfectly synchronized lasers for the fast heating and compression indicated that it may be possible to use longer pulses $(>10 \mathrm{ps})$ to achieve ignition.

Work at the RAL this year has concentrated on an important objective related to these new observations, namely confirmation of the results at an independent laboratory using similar laser conditions and, perhaps more importantly, understanding electron and ion energy transport under these conditions. To minimize changes between the experiments at the RAL and ILE, hollow deuterated plastic shells with similar diameter $(500 \mu \mathrm{m})$ and thickness $(6 \mu \mathrm{m})$ were used.

\section{EXPERIMENTAL CONFIGURATION}

The target area and chamber were configured so that the 100 TW beam was coupled to the nanosecond drive beams in 6-beam cubic symmetry. The main difference between the experiments at the two laboratories was the color of the main drive beams (527 nm for ILE, $1054 \mathrm{~nm}$ for this experiment at RAL) to maintain the incident energy on target.

The requirement was for highly synchronized coupling of the nanosecond drive beams to the 100 TW beam line to eliminate uncertainty in timing between the heating pulse and stagnation of the implosion. The normal operational 


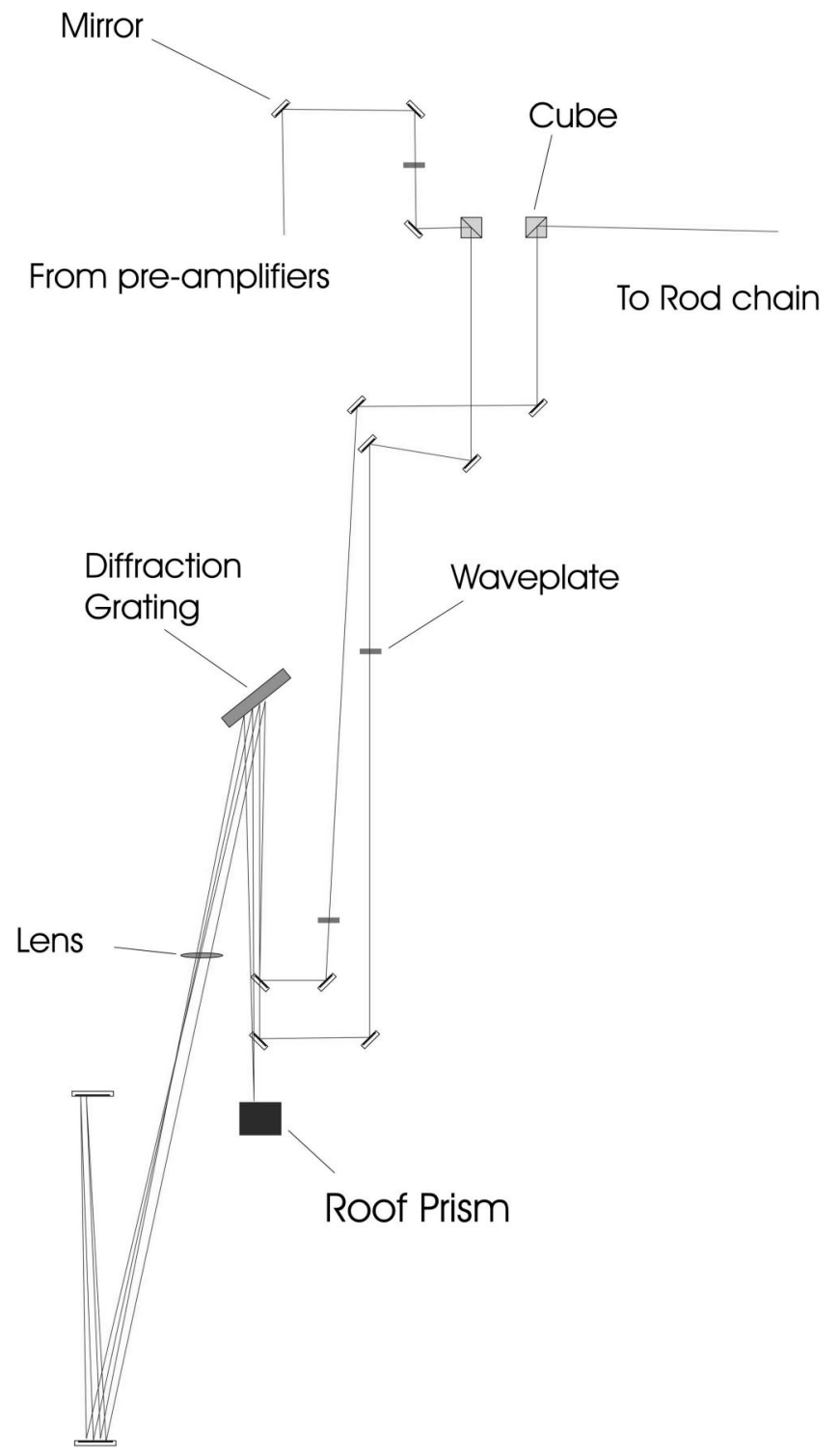

FIG. 1. Outline of the stretcher configuration to provide absolutely synchronized nanosecond and picosecond laser pulses to target.

mode for the VULCAN laser $^{5}$ is to use a Single Longitudinal Mode (SLM) laser oscillator to generate pulses of nanosecond duration. Pulse synchronization is normally achieved using the rf from the SLM oscillator to drive the Pockels cells in the two parallel rod-amplifier chains. This normally generates $\sim 150 \mathrm{ps}$ of jitter in the timing between the long and short pulses that was not, in this case, considered acceptable. The solution was to split single pulses generated by the short pulse oscillator into two halves. This was performed after the short pulse had been stretched to $300 \mathrm{ps}$ duration and amplified in two single pass preamplifiers. An additional stretcher was designed to increase the normal stretched pulse length from 300 ps to 900 ps for one of these two beams and is illustrated in Fig. 1. This additional double-pass stretcher configuration used a 1470 line per millimeter grating and a $60 \mathrm{~cm}$ focal length lens. The pulse was then amplified in a third preamplifier before being introduced into the Vulcan rod chain to generate the six long pulse drive beams.

After amplification through the entire laser system, each

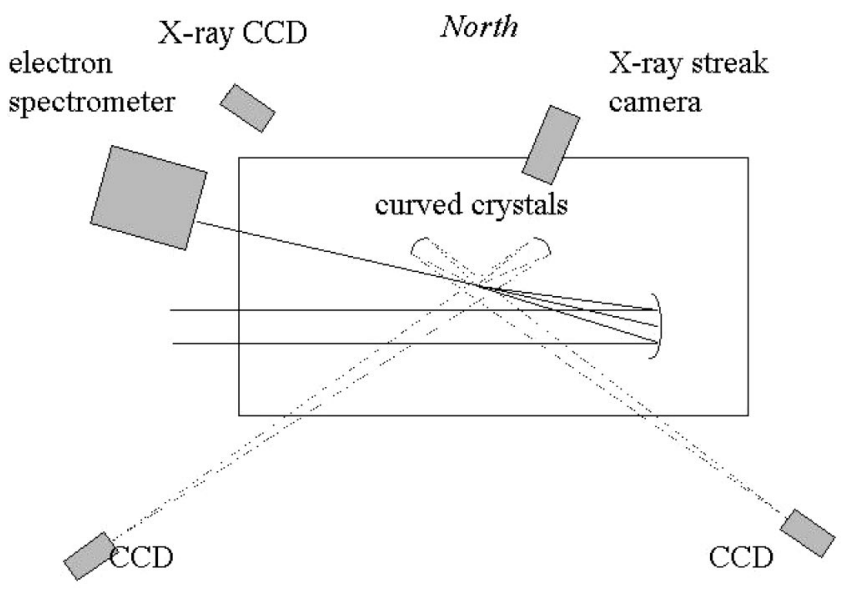

FIG. 2. Illustration of the plasma diagnostics.

of the six $900 \mathrm{ps}, 10.8 \mathrm{~cm}$ diam beams delivered $150 \mathrm{~J}$ to the target via an f 2.5 lens. Four of these six drive beams were incident onto target at $45^{\circ}$ to the horizontal, directly along the east west axis. The other two beams were located on the horizontal plane in the north-south axis. Phase zone plates, that produced $500 \mu \mathrm{m}$ focal spots, were used in initial implosions. It was found that the energy coupling efficiency to the implosion was poor when these phase plates were used. In the results reported below, the $\mathrm{f} 2.5$ beams were focused with their marginal rays tangential to the sphere without phase plates.

The $100 \mathrm{TW}$ beam was introduced in the horizontal plane at $15^{\circ}$ from the east-west axis. This final size of this beam after amplification through the outer arm of the VULCAN laser and after compression was $20 \mathrm{~cm} \times 11 \mathrm{~cm}$. The laser delivered up to $80 \mathrm{~J}$ to target with pulses in the range $0.8 \mathrm{ps}-1.1 \mathrm{ps}$. The beam was focused using a $60 \mathrm{~cm}$ off-axis parabola. An equivalent plane monitor indicated that $30 \%$ of the laser energy was located within a $10 \mu \mathrm{m}$ focal spot. The intensity contrast ratio has been measured previously to be $10^{-7}$. $^{-1}$

The plasma diagnostics are illustrated in Fig. 2 and included current mode time-of-flight neutron detectors set up outside the chamber. The $\mathrm{x}$-ray pinhole camera was filtered by $25 \mu \mathrm{m}$ thick $\mathrm{Be}$, so that it was sensitive to photons above $1 \mathrm{keV}$. The detector was KODAK DEF x-ray film. The camera had a magnification of $\times 10$. The pinhole size was $10 \mu \mathrm{m}$ and was set in $7 \mu \mathrm{m}$ thick Pt substrate. A KENTECH x-ray streak camera was coupled to $10 \mu \mathrm{m}$ slit that was placed 15 $\mathrm{cm}$ from the target at a magnification of $\times 5$ to provide a one-dimensional streaked image of the implosion.

An electron spectrometer was employed directly along the $100 \mathrm{TW}$ laser axis from the off-axis parabolic mirror on the horizontal plane to detect the electron spectra generated by the ultraintense laser pulse. The spectrometer, illustrated in Fig. 3, consisted of a pair of permanent magnets with dimensions $15 \mathrm{~cm} \times 3 \mathrm{~cm}$ separated by $3 \mathrm{~cm}$. These produce a magnetic field in the gap of $0.14 \mathrm{~T}$. The magnetic field deflected electrons onto a FUJIFILM BAS-SR 2025 image plate (diameter $20 \mathrm{~cm} \times 25 \mathrm{~cm}$ ) located on the top of the magnet structure in Fig. 3. The image plates work by photo- 


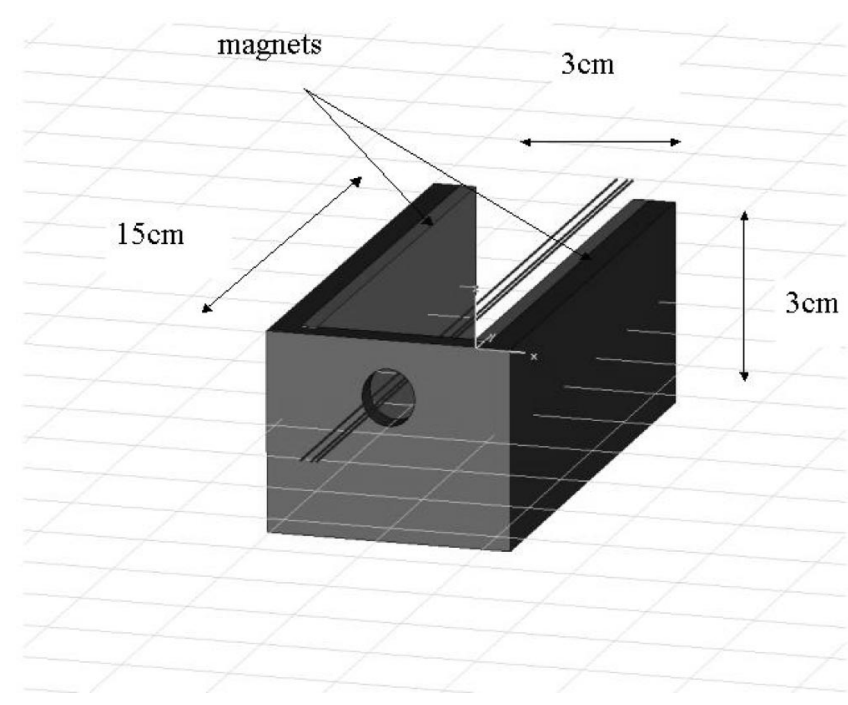

FIG. 3. Outline of the electron spectrometer.

stimulated luminescence, i.e., ionizing particles induce electrons in the substrate to occupy metastable levels that are de-excited by longer wavelength laser radiation used in the reader. ${ }^{6}$ The information was digitally recorded using a FUJIFILM BAS-1800II (Ref. 8) reader after $80 \mathrm{~min}$. The spectrometer was located $1 \mathrm{~m}$ from the target and was capable of detecting electrons in the energy range $0.1-40 \mathrm{MeV}$.

Two spherically curved crystals were used for x-ray imaging. ${ }^{7}$ The first was used for backlighting in the initial phase of the experiment and the second was used for direct $K_{\alpha}$ imaging of $\mathrm{Cu}$ impurity $(h \nu=8.05 \mathrm{keV})$ doped into the shell. Ti $K_{\alpha}$ radiation $(h \nu=4.51 \mathrm{keV})$ was used for the X-ray radiographic studies. The areal density was obtained by measurement of the attenuation of the monochromatic $\mathrm{x}$ rays through the compressed matter. Snapshots were made at different times on consecutive shots to determine the peak stagnation time of the implosion. In this case, the $100 \mathrm{TW}$ beam was displaced by several millimeters to produce a $1 \mathrm{~mm}$ $\times 0.5 \mathrm{~mm}$ focal spot on a $25 \mu \mathrm{m}$ thick Ti foil giving an intensity on target of $1.6 \times 10^{16} \mathrm{~W} \mathrm{~cm}^{-2}$. The $K_{\alpha}$ radiation generated was imaged using a spherically curved crystal onto a Princeton Instruments 16-bit x-ray charge-couple device (CCD) camera.

A single photon counting Spectral Instruments $\mathrm{x}$-ray CCD camera was fielded to record the spectra of the $K_{\alpha}$ backlighter and give the absolute $K_{\alpha}$ yield. A $2000 \times 2000$ pixel back-thinned CCD chip with a pixel size of $13 \mu \mathrm{m}$ was used and the images were recorded with 16-bit resolution. Figure 4 shows a typical Ti $K_{\alpha}$ spectrum under these conditions. The spectral resolution of the single photon counting CCD camera is not sufficient to resolve $K_{\alpha}$ the spectral line, the linewidth shown in the graph is dominated by the instrumental broadening. Using a detection efficiency of $50 \%$, which is typical for $\mathrm{x}$-ray $\mathrm{CCD}$ cameras in this configuration, ${ }^{8}$ a conversion efficiency on the order of 1 $\times 10^{-5}$ from laser energy into $K_{\alpha}$ photons is inferred from the spectrum.

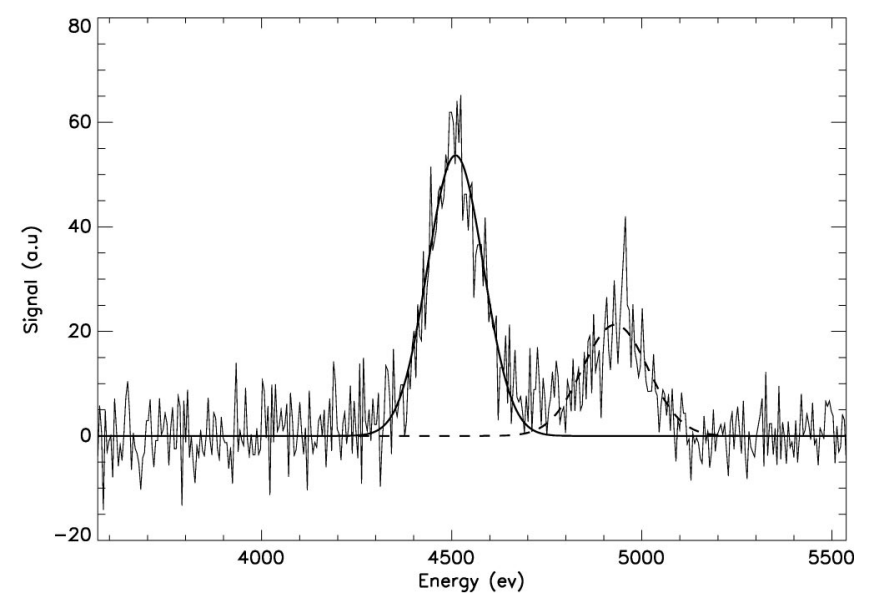

FIG. 4. Ti $K$-shell spectrum from the single photon counting x-ray CCD camera, with fits of the $K_{\alpha}$ line at $4510 \mathrm{eV}$ (solid line) and the $K_{\beta}$ line at $4930 \mathrm{eV}$ (dashed line).

\section{IMPLOSION DYNAMICS}

Shells contained 0.7 at $\%$ of $\mathrm{Cu}$. The shells had a diameter of $486 \mu \mathrm{m}$, a wall thickness of $6 \mu \mathrm{m}$ and an initial density of $1.3 \mathrm{~g} \mathrm{~cm}^{-3}$. Identical shells were mounted on $\mathrm{Au}$ cones as illustrated in Fig. 5 and were manufactured by the target fabrication facility at General Atomics. The inner edge of the cone had an opening half angle of $13^{\circ}$. The outer edge had a half angle of $22^{\circ}$. A step on the Au cone located the shell in position such that the cone tip was $40 \mu \mathrm{m}$ from the stagnation point.

To determine stagnation time, identical diameter and thickness spherical shells were irradiated with the $0.9 \mathrm{~kJ}$, nanosecond drive laser pulse. Significant decompression of the shell occurs in the coasting phase after the drive pulse is finished. Figure 6 shows the radius of the shell from the radiographs taken after different times. Plotted here is the radius-time trajectory calculated using the one-dimensional (1D) Lagrangian hydrodynamic code MEDUSA. ${ }^{9}$ Stagnation occurs at $3.1 \mathrm{~ns}$ after the peak of the drive pulse. Comparison of the measured X-ray absorption with opacities calculated using the LASNEX 1D hydrocode indicate that peak compressed densities of $4 \mathrm{~g} \mathrm{~cm}^{-3}$ were achieved. ${ }^{10}$ The next step

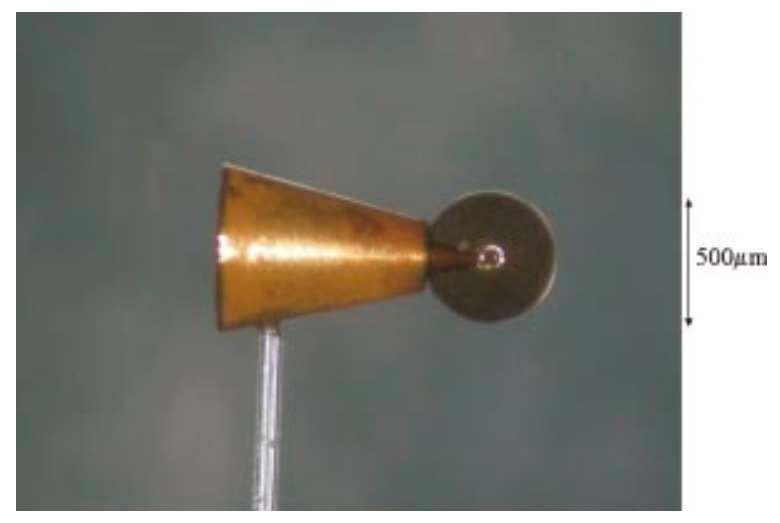

FIG. 5. (Color) Picture of a typical CD shell attached to a hollow Au cone. The tip of the cone is located by the collar at $40 \mu \mathrm{m}$ from the stagnation point. The end wall thickness of Au was $5 \mu \mathrm{m}$. 


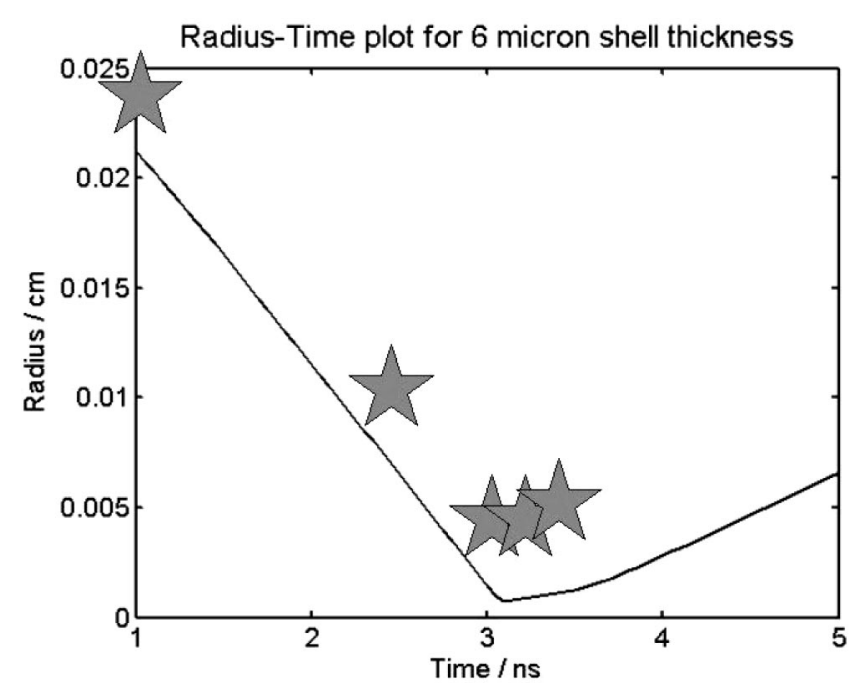

FIG. 6. Radius-time plot from the 1 D MEDUSA code and the measured diameter of the shell at different times.

was to radiograph a cone-shell implosion at peak compression. The image demonstrates that the $100 \mu \mathrm{m}$ diam compressed plasma is formed close the tip of the cone, corresponding to a peak areal density of $40 \mathrm{mg} \mathrm{cm}^{-2}$. The details of these measurements will be published shortly elsewhere. ${ }^{11}$

Comparisons of the compressed density that can be achieved in hydrodynamic simulations with and without radiation transport included indicates that this process plays a central role in limiting the compressed density. The MEDUSA simulations that do not include radiation predict, for example, compressed densities of $350 \mathrm{~g} \mathrm{~cm}^{-3}$ and $\sim 10 \mu \mathrm{m}$ core diameter, even when the absorbed energy is adjusted to match to the measured stagnation time. Similar results were obtained from the LASNEX code without radiation transport, but when this was included, the predicted density was significantly reduced to $9 \mathrm{~g} \mathrm{~cm}^{-3}$ with $80 \mu \mathrm{m}$ core diameter. The measured and calculated densities are therefore in reasonable agreement when radiation transport is taken into account.

(a)

(b)

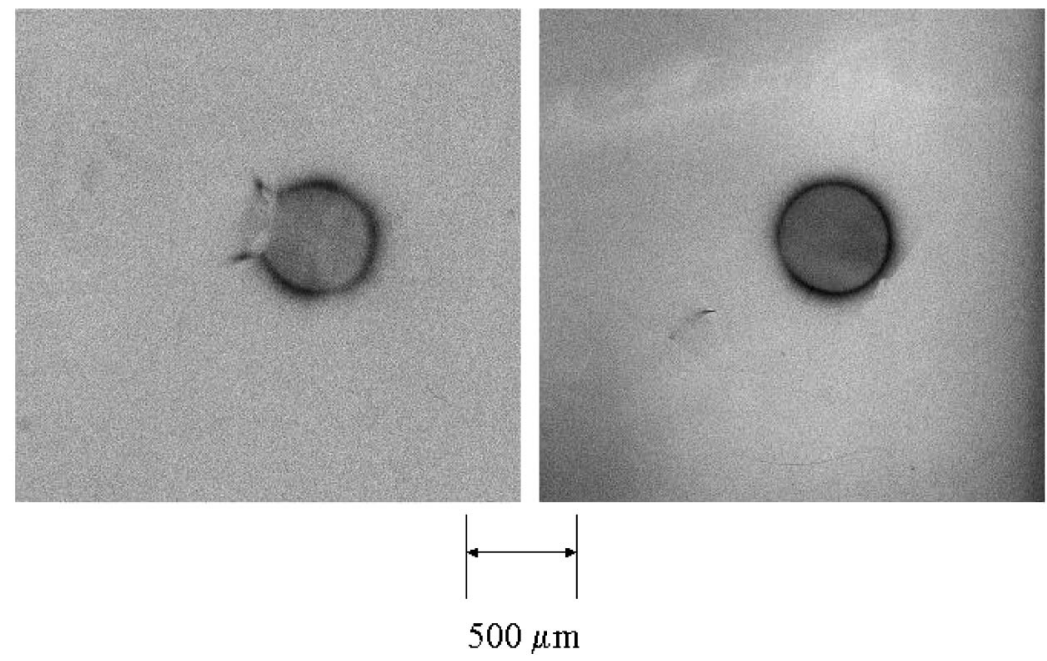

Radiative transport allows $\mathrm{x}$ rays from the laser deposition region to penetrate and preheat the dense shell, making the shell much less compressible at stagnation. Whether or not radiative preheat is included in the simulations, the shell at stagnation is well off the degenerate adiabat, but the entropy differences are large between the two cases. With radiation transport included, the entropy of highest density at peak $\rho R$ is $\sim 3.6 \times 10^{8} \mathrm{~J} / \mathrm{g} / \mathrm{keV}$. Without the transport included, the entropy is $\sim 2.0 \times 10^{8} \mathrm{~J} / \mathrm{g} / \mathrm{keV}$. The pressure for a given density is a factor of about 6.3 higher with radiation (or, put the other way, without radiation the density is a factor of 3.1 greater for a given pressure). Moreover the capsule without radiation reaches a much higher stagnation pressure, because what is being held constant is the amount of work the imploding shell's kinetic energy can do: so a relatively high pressure at a given volume means the kinetic energy of the shell with radiation is all used up before the volume changes much.

Figure 7 shows two x-ray images of the implosion with and without the cone insert. There is no $\mathrm{x}$-ray emission from the core visible. This indicates that the residual thermal energy is low $(\sim 50 \mathrm{eV})$ at stagnation. These results confirm that there was little chance of diagnosing the implosion by neutron spectroscopy-as the temperature (even with increase due to the short pulse heating) is too low for any significant number of thermonuclear reactions to occur.

\section{HEATING WITH 1 PS AND 10 PS PULSES}

The measurements above confirm that the compressed density is $4 \mathrm{~g} \mathrm{~cm}^{-3}$ and the areal density is $40 \mathrm{mg} \mathrm{cm}^{-2}$. These conditions are somewhat lower than those obtained at earlier at Osaka University which had $2.4 \mathrm{~kJ}$ of $527 \mathrm{~nm}$ laser drive energy available for compression. There the compressed density was $50-70 \mathrm{~g} \mathrm{~cm}^{-3}$ and the areal density was $200 \mathrm{mg} \mathrm{cm}^{-2}$. The latter conditions more closely match the range of $\mathrm{MeV}$ electrons generated by the 100 TW heating beam.

In an attempt to match the range of fast electrons to realized compressed density in the experiment, the pulse du-
FIG. 7. Typical x-ray images ( $>1.5 \mathrm{keV})$ of (a) a cone/ shell implosion and (b) a complete spherical shell. 


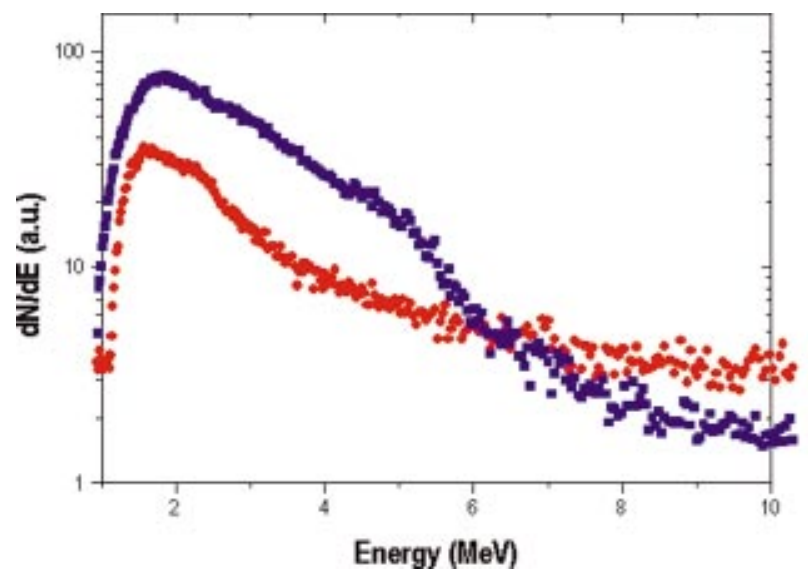

FIG. 8. (Color) Electron spectra taken with $10 \mathrm{ps}$ irradiation of a $25 \mu \mathrm{m}$ thick $\mathrm{Cu}$ foil (blue) and a stand-alone cone (red). No electron spectra were observed with cone/shell implosions at stagnation.

ration of the short pulse beam was increased to $10 \mathrm{ps,} \mathrm{pro-}$ viding an intensity on target of $2.5 \times 10^{18} \mathrm{~W} \mathrm{~cm}^{-2}$. Figure 8 shows two electron spectra obtained under these conditions. The first is when the now 10 TW beam was focused onto a single $\mathrm{Cu}$ foil at this maximum intensity. The second was for a hollow Au cone without an implosion. It can be seen that the number of electrons differs by no more than a factor of 3 in the range 1-10 MeV. There was no electron signal in the spectrometer when the $10 \mathrm{ps}$ duration heating beam was introduced into a cone shell implosion at stagnation. There was also no noticeable increase $\mathrm{x}$-ray emission observed in the $\mathrm{x}$-ray pinhole images.

Figure 9 shows two electron spectra taken when the pulse duration was minimized to 1 ps duration. The first is when the $100 \mathrm{TW}$ beam was focused onto a single $\mathrm{Cu}$ foil at maximum intensity. The electron distribution for the foil case is well described by a simple $\exp (-E / k T)$ Maxwellian distribution with $k T=4 \mathrm{MeV}$. This is reasonable agreement with a ponderomotive potential for an intensity on target of 1 $\times 10^{20} \mathrm{~W} \mathrm{~cm}^{-2}$, suggesting there may be some self-focusing

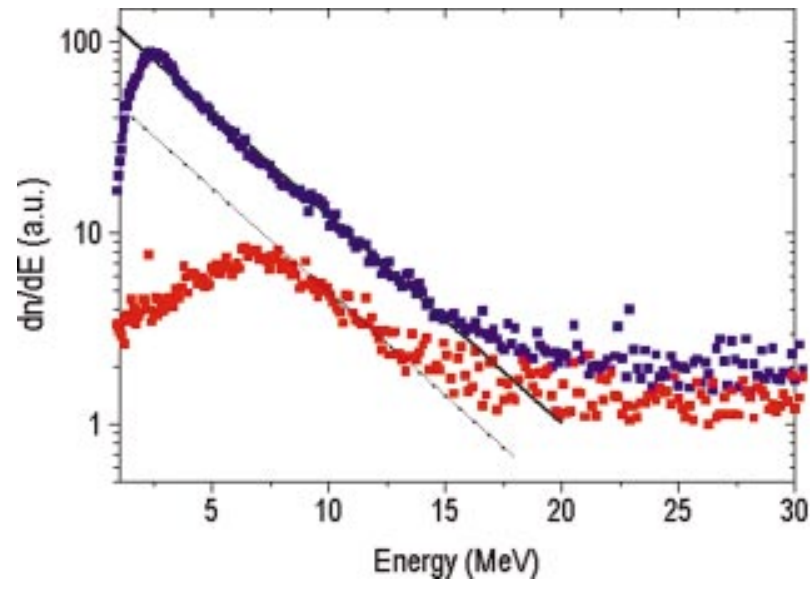

FIG. 9. (Color) Electron spectra taken with 1 ps irradiation of a $25 \mu \mathrm{m}$ thick $\mathrm{Cu}$ foil (blue) and a cone/shell implosion at stagnation (red). Notice that there is a significant reduction in $2.5 \mathrm{MeV}$ electrons reaching the spectrometer in the latter case. Both distributions can be fitted with a $\exp (-E / k T)$ distribution with $k T=4 \mathrm{MeV}$ (see the dotted black lines in both spectra).

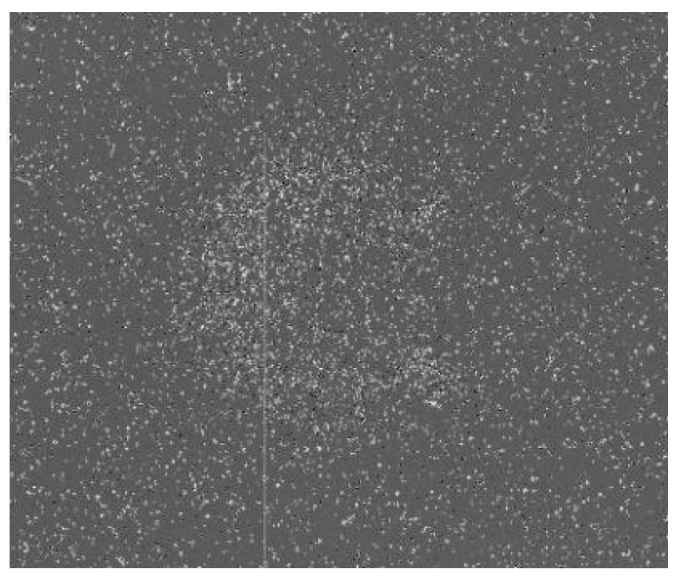

$500 \mu \mathrm{m}$

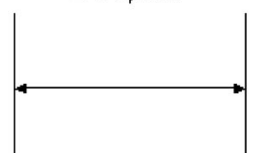

FIG. 10. Two-dimensional $\mathrm{Cu} K_{\alpha}$ image of the implosion and heating with 1 ps irradiation. Notice the limb brightening around the original target position caused by hot electrons generated during the nanosecond duration laser drive pulse. There is no visible core emission, and this sets a lower limit to the energy transferred to the compressed plasma from the 100 TW pulse.

due to hole-boring occurring during the interaction. The second spectrum in Fig. 9 (red) was obtained during a cone/shell implosion at stagnation with a $1 \mathrm{ps}$ duration heating pulse. It is clearly seen, however, that electrons below $7 \mathrm{MeV}$ are severely attenuated. The number of electrons at $2.5 \mathrm{MeV}$ is reduced by a factor of 8 , taking into account the factor of 3 difference between foil and stand-alone cone that is seen again in the electron distribution above $7 \mathrm{MeV}$.

Figure 10 shows a typical $K_{\alpha}$ image obtained with the spherical crystal imager for integrated implosion/heating shots using $1 \mathrm{ps}$ irradiation. A similar $500 \mu \mathrm{m}$ diam faint image was observed corresponding to emission from the $\mathrm{Cu}-$ doped shell during the early stage of the implosion, with either 1 or $10 \mathrm{ps}$ short pulse heating. This emission is due to hot electrons generated during the nanosecond laser-plasma interaction. No core emission was observed from the $1 \mathrm{ps}$ or $10 \mathrm{ps}$ irradiation and this sets an upper limit to the fraction of the total imaged $K_{\alpha}$ yield which is from the core. Absolute calibration of the image intensity was obtained using a solid $\mathrm{Cu}$ target. The photon counting CCD spectrometer measurement of the absolute $K_{\alpha}$ yield and the corresponding image provided the absolute calibration. The upper limit on energy transferred to the fast electrons from the 1 ps heating pulse, was thus estimated from the upper limit $K_{\alpha}$ yield using a model discussed elsewhere ${ }^{12}$ to be $\sim 1 \mathrm{~mJ}$.

\section{DISCUSSION}

The following discussion is based upon the assumption that the data from the absolutely calibrated spherically curved crystal spectrometer image gives a true record of the total $K_{\alpha}$ emission. For the implosion shots we have recently found a discrepancy between the absolute $\mathrm{Cu} K_{\alpha}$ yield obtained with the single photon counting $\mathrm{CCD}$ and that ob- 
tained with the spherically curved crystal spectrometer. The former indicates $60 \times$ more yield. The origin of this discrepancy is possibly due to a frequency shift of the $K_{\alpha}$ emission exceeding the bandwidth of the crystal. This opens up two possible different interpretations of the data. One is simply a 60 -fold scale up in the absolute brightness of the $K_{\alpha}$ image and the conclusions from that brightness. Another is that the frequency shifted $K_{\alpha}$ emission is predominantly from the short pulse interaction with the core and that the image does not show this. These alternative possibilities are the subject of current study but are not considered further here.

The lack of any increase in $\mathrm{x}$-ray emission in the pinhole images, the absence of hot electrons in the electron spectrometer and of any core emission in the $K_{\alpha}$ image formed from the spherically curved crystal spectrometer suggests that substantial hydrodynamic motion might have occurred under $10 \mathrm{ps}$ irradiation conditions at stagnation. Of course, the electron source could still be different between a solid foil and an imploded cone, due to the different plasma scalelength in the interaction region (that is, unfortunately, not easily measurable). Also, electron spectra measured some distance from the target may not necessarily reflect the electron distribution inside the dense plasma due to space-charge electric fields. Hot electrons, for example, are known to accelerate ions to multi-MeV energies due to the sheath acceleration mechanism-and it is known that the maximum potential that these ions experience depends upon initial plasma parameters, such as the density scale-length.

However, when magnetic-field instability effects are neglected, it has been shown that electron energy transport can be separated into five distinct temporal regimes or eras. ${ }^{13}$ The first is the transient J.E regime- the hot electrons move relatively unimpaired by the electric field set-up to draw the return current and where the background electrons are heated by the J.E effect. This is followed by the J.E heating regime - the hot electrons are slowed down by the electric field determined from the resistive background electrons. The next era is the J.E drag transition regime-drag heating dominates the temperature equation and the density scalelength is determined by the cold electron resistivity. This is followed by the J.E drag regime itself-again drag heating dominates the temperature equation, but here the density scale-length is now dominated by the hot electron collisionality. Finally, the diffusive regime is entered - the temperature gradient becomes so large that thermal diffusion carries the deposited energy into the target. In this scenario, the small numbers of electrons that escape the target using the 100 TW pulse (compared with the total fast electron population) can probably only do so during the initial transient J.E regime - in which case, they should contain information on the initial distribution. This is clearly seen, for example, in the temperatures deduced from electron spectra by Malka and Miquel in earlier studies ${ }^{14}$ and the good agreement between those observations and theory.

Bearing these arguments in mind in the following discussion, four possible reasons for a reduction in the number of $2.5 \mathrm{MeV}$ electrons reaching the spectrometer for $1 \mathrm{ps}$ irradiation at stagnation will be presented: increased small angled scattering in the compressed plasma; beam break-up at $n_{c} / 2$; anomalous resistivity and transport inhibition in a plasma with a longer scale-length. Let us begin with increased small angle scattering in the compressed plasmathis would increase the cone angle of the escaping electrons. Since the electron spectrometer subtends only a small solid angle, this effect would reduce the numbers of $\mathrm{MeV}$ electrons being detected. Suppose that the fast electrons are emitted in a $\sim 20^{\circ}$ cone angle before they traversed the compressed plasma, an angle consistent with previous measurements. ${ }^{12}$ This would correspond to a solid angle of $\sim \pi / 4$ sr. The reduction in electron numbers can only be accounted for provided that $50 \%$ of the $2.5 \mathrm{MeV}$ electrons were scattered isotropically into $4 \pi$ sr, which appear unlikely.

To look at this question of the transport of $\mathrm{MeV}$ electrons in further detail, two-dimensional computer simulations using Mission Research Corporations' LSP code were undertaken. ${ }^{15}$ The LSP simulations, whose details will be published elsewhere, ${ }^{16}$ were performed with an injected electron beam into the deuterated plastic plasma whose density was varied between $1 \mathrm{~g} \mathrm{~cm}^{-3}$ and $4 \mathrm{~g} \mathrm{~cm}^{-3}$. The code treated the background electrons as a fluid, whereas the injected electrons were treated as particles. The fluid background had a perfect gas equation of state and Spitzer transport coefficients. The code assumed a parallel electron beam temperature of $k T=2.5 \mathrm{MeV}$ and a transverse temperature of $k T$ $=300 \mathrm{keV}$. Figure 11 shows the results of the simulations. They indicate that a higher background density promotes the collimation of the injected electron beam, due to the competition between Weibel and whole beam self-focusing. However, it also reduces the peak background temperature reached for these higher densities. This is due to the fact that collisional heating is proportional to density, the resistive heating is independent of density and the heat capacity proportional to density. These simulations indicate that it may be difficult to resistively heat high density $\left(200 \mathrm{~g} \mathrm{~cm}^{-3}\right)$ plasmas for fast ignition. What they do indicate is that if the electron beam is more collimated inside the overdense region, then the electron spectrometer might be expected to register more electrons escaping the target, not fewer. Of course, it is conceivable that electrons that escape the target could miss the entrance to the electron spectrometer if the target is misaligned in some manner, but great care was taken to ensure that the cone/shell implosions were aligned correctly. This is an unlikely cause of the reduction in electron numbers.

Another possible reason for the reduction of $2.5 \mathrm{MeV}$ electrons is beam-breakup at $0.5 n_{c}$. It is interesting to note that the hot electron distribution for the $25 \mu \mathrm{m}$ thick $\mathrm{Cu}$ foil in Fig. 10 can be fitted using an approximation to a twotemperature relativistic Maxwellian distribution given by

$$
\begin{aligned}
\frac{d N}{d E} \approx & 100 E^{2} \exp (-E / 1.25 \mathrm{MeV})+E^{2} \\
& \times \exp (-E / 3.75 \mathrm{MeV}) .
\end{aligned}
$$

This would indicate predominantly ponderomotive acceleration into the target with a small amount of plasma wave acceleration in the coronal plasma. The electron spectra from 


\section{Increasing background density changes the competition between Weibel (filamentation) and whole beam focussing.}

\section{Sequence $\rho=1,2,4 \mathrm{~g} \mathrm{~cm}^{-3}$}

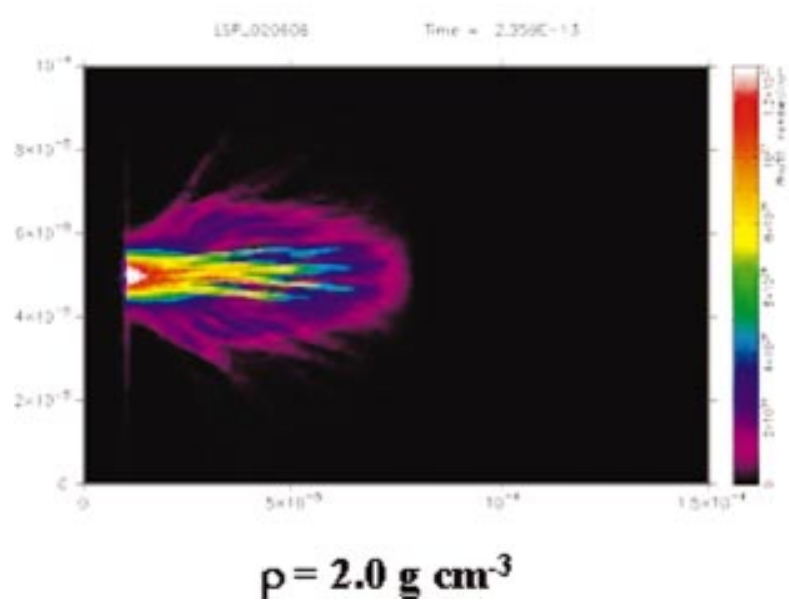

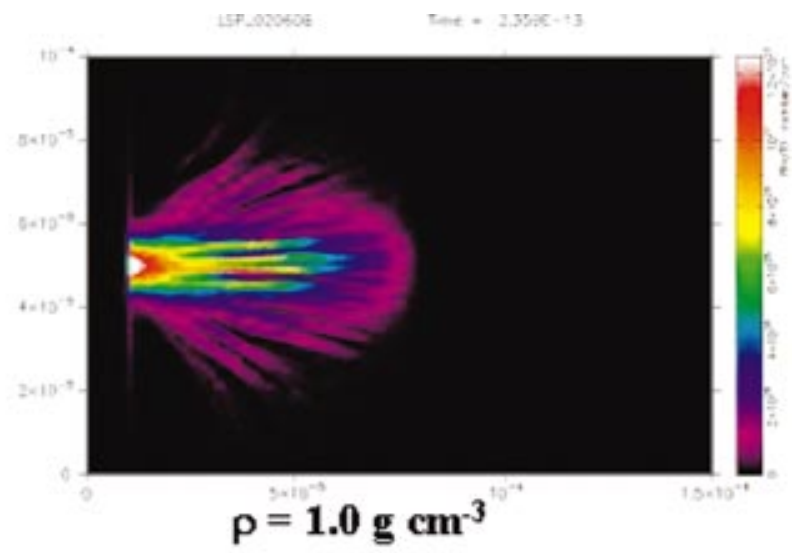

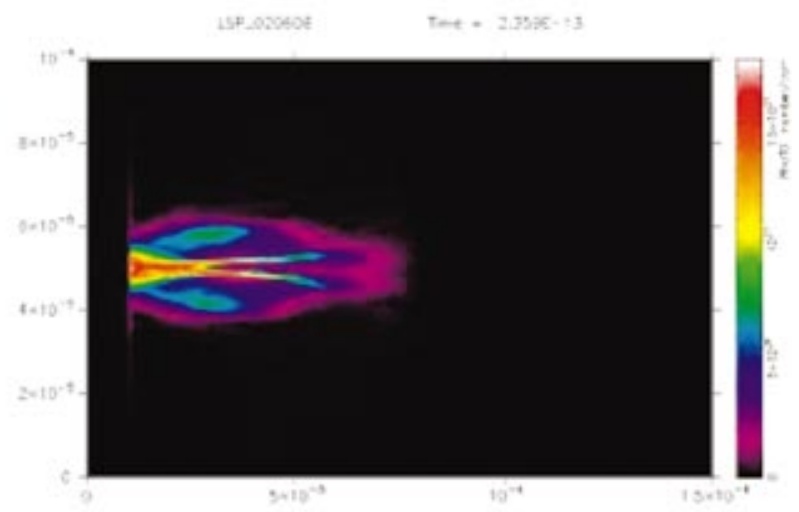

$\mathrm{p}=4.0 \mathrm{~g} \mathrm{~cm}^{3}$

FIG. 11. (Color) LSP simulations of electron beam density taken at $235 \mathrm{fs}$ into the pulse for three different densities. The mesh resolution is $400 \times 600$ cells with 0.25 micron size. The target is $150 \mu \mathrm{m} \times 100 \mu \mathrm{m}$.

the cone/shell implosion spectrum in Fig. 9, on the other hand, can be fitted using a single temperature distribution given by

$$
\frac{d N}{d E} \approx E^{2} \exp (-E / 3.25 \mathrm{MeV}) .
$$

This would suggest that all the ponderomotive electrons are missing and, if this were the case, none of the incident laser energy would have reached the critical density surface. Filamentation of an ultraintense laser pulse has been observed in experiments performed at the ILE Osaka University during the interaction of ultraintense laser pulses with plasmas with long scale-lengths. ${ }^{17}$ This instability occurred when the laser focus was beyond the position of the critical density surface, corresponding to plasma densities between $0.5 n_{c}$ and $0.8 n_{c}$. On the other hand, simultaneous optical measurements indicated little difference in energy backscattered under these conditions compared to when the laser was focused directly on the critical surface. It therefore appears unlikely that filamentation would have prevented all the energy reaching the critical density surface in this case.

Another possibility is that anomalous resistivity is playing an important role in these interactions. The LSP simulations assume Spitzer resistivity for the background electrons. This is calculated assuming that the small angle scattering events are single electron-electron collisions that are summed over the Maxwellian distribution. When the mag- netic field is large enough, for example in the presence of fine-scale filamentary structures generated by the magnetic Weibel or tearing mode instabilities, the Lamor radius of the colder electrons in the return current can be very small. In this situation, multiple scattering events can occur with the same electron or ion. This quickly leads to the onset of turbulence and results in a substantial increase in the plasma resistivity. Sentoku et al., for example, has recently calculated that there is as much as $10^{3}$ increase in resistivity compared with classical Spitzer values for PW laser-plasma interactions. ${ }^{18}$ It is interesting to note that the absence of $\mathrm{Cu} K_{\alpha}$ emission from the core in Fig. 10 indicated that the energy transferred to the compressed plasma is close to the detection threshold, i.e., $1 \mathrm{~mJ}$. It appears from these observations that the fast electron energy did not reach the compressed core plasma and that, as a result, this mechanism is an unlikely explanation, at least for these conditions.

There is one further possibility, that is transport inhibition in overdense plasmas due to the generation of a larger scale-length plasma formed inside the cone. The larger scalelength plasma could be generated by fast electrons generated during the nanosecond laser-plasma interaction penetrating the end wall of the cone and generating a plasma on the inner surface. That these electrons were generated was confirmed by images of the $\mathrm{Cu} K_{\alpha}$ radiation from the doped $\mathrm{CD}$ shell that were emitted during the acceleration phase of the implosion presented in Fig. 11. This relatively cold plasma would 
have had $\sim 3 \mathrm{~ns}$ to expand into the vacuum before the short pulse laser energy was delivered. The electric field set up to draw the return current is given by $\mathbf{E}=\eta \mathbf{J}$ (from the generalized Ohm's law). From this, the plasma resistivity $\eta$ is, to a first approximation, independent of density. However, the return current electrons have to drawn from the background plasma. If the plasma has a larger scale-length, then the electric field must extend over a much larger distance than a plasma with a smaller scale-length in order to supply the return current. This has the effect of reducing fast electron transport into the target, since the fast electrons are slowed down by the extended electric field. In effect, the fast electrons are "bottled up" in the extended plasma between critical and solid. This effect, confirmed by LSP simulations, has been used to explain recently observed reduced heating of buried Al signature layers using time-resolved $\mathrm{x}$-ray emission spectroscopy from the PW laser arm of the VULCAN laser facility. ${ }^{19}$

It would appear that the key to efficient energy transport in cone/shell implosions is control of the density scale-length of the PW laser interaction process.

\section{SUMMARY}

Cone shell implosions have been performed on the VULCAN laser using the full 0.9-kJ output of the system. $\mathrm{X}$-ray radiographic studies have indicated that compressed densities of $4 \mathrm{~g} \mathrm{~cm}^{-3}$ and areal densities of $40 \mathrm{mg} \mathrm{cm}^{-2}$ were achieved. X-radiation preheat, combined with lower ablation pressures, indicate that infrared drive is unlikely to be useful in full-scale direct drive ignition designs, due to the limited compressed densities that can be achieved, at least for designs requiring sub-MJ drive energy. Nearly an order of magnitude reduction in the number of $2.5 \mathrm{MeV}$ electrons escaping the target to the electron spectrometer was observed when 1 ps pulses were used. Computer simulations show that the higher density of the compressed plasma promotes more collimated flows, but at the cost of reduced background heating. They also suggest that it is difficult to resistively heat higher density $\left(200 \mathrm{~g} \mathrm{~cm}^{-3}\right)$ plasmas for fast ignition, when classical Spitzer resistivity is used for the background electron fluid. It is likely that hot electrons, generated during the nanosecond drive beam, generated a larger preformed plasma inside the cone that prevented efficient energy transport into the compressed core. It therefore appears from these observations that control of the density scale-length of the heating beam may be essential for efficient energy coupling and hot spark formation. These observations have implications for the experiments using the next generation of PW lasers now under construction.

\section{ACKNOWLEDGMENTS}

The authors gratefully acknowledge the assistance of all the staff at the Central Laser Facility and Jörg Schreiber.
This work was supported by the United Kingdom Engineering and Physical Sciences Research Council/Ministry of Defense Grant No. GR/R16778. Japanese colleagues acknowledge support from the Japan Society for the Promotion of Science. For Lawrence Livermore National Laboratory (LLNL) authors, this work was performed under the auspices of the United States Department of Energy by the LLNL under Contract No. W-7405-ENG-48.

${ }^{1}$ M. Tabak, J. Hammer, M. E. Glinsky, W. L. Kruer, S. C. Wilks, J. Woodworth, E. M. Campbell, M. D. Perry, and R. J. Mason, Phys. Plasmas 1, 1626 (1994).

${ }^{2}$ P. A. Norreys, R. Allott, R. J. Clarke, J. Collier, D. Neely, S. J. Rose, M. Zepf, M. Santala, A. R. Bell, K. Krushelnick, A. E. Dangor, N. C. Woolsey, R. G. Evans, H. Habara, T. Norimatsu, and R. Kodama, Phys. Plasmas 7, 3721 (2000).

${ }^{3}$ R. Kodama, P. A. Norreys, K. Mima, A. E. Dangor, R. G. Evans, H. Fujita, Y. Kitagawa, K. Krushelnick, T. Miyakoshi, N. Miyanaga, T. Norimatsu, S. J. Rose, T. Shozaki, K. Shigemori, A. Sunahara, M. Tampo, K. A. Tanaka, Y. Toyama, T. Yamanaka, and M. Zepf, Nature (London) 412, 798 (2001).

${ }^{4}$ R. Kodama, H. Shiraga, Y. Toyama, S. Fujioka, H. Azechi, T. Jitsuno, Y. Kitagawa, K. M. Krushelnick, K. L. Lancaster, K. Mima, K. Nagai, M. Nakai, H. Nishimura, T. Norimatsu, P. A. Norreys, S. Sakabe, K. A. Tanaka, A. Youssef, M. Zepf, T. Yamanaka, and R. Kodama, Nature (London) 418, 922 (2002).

${ }^{5}$ C. N. Danson, J. Collier, D. Neely, L. Barzanti, A. Damerell, C. B. Edwards, M. H. R. Hutchinson, M. H. Key, P. A. Norreys, D. A. Pepler, I. N. Ross, P. F. Taday, W. T. Toner, M. Trentelman, F. N. Walsh, T. B. Winstone, and R. W. W. Wyatt, J. Mod. Opt. 45, 1653 (1998).

${ }^{6}$ J. Miyahara, K. Takahashi, Y. Amemiya, N. Kamiya, and Y. Satow, Nucl. Instrum. Methods Phys. Res. A 246, 572 (1986).

${ }^{7}$ J. A. Koch, Y. Aglitskiy, C. Brown, T. Cowan, R. Freeman, S. Hatchett, G. Holland, M. Key, A. MacKinnon, J. Seely, R. Snavely, and R. Stephens, Rev. Sci. Instrum. 74, 2130 (2003).

${ }^{8}$ M. Nishiuchi, K. Koyama, T. Tsuru, H. Awaki, H. Tomida, K. Hamaguchi, and H. Tsunemi, Proc. SPIE 3445, 268 (1998).

${ }^{9}$ J. P. Christiansen, D. E. T. F. Ashby, and K. V. Roberts, Comput. Phys. Commun. 7, 271 (1974).

${ }^{10}$ G. B. Zimmerman, Comments Plasma Phys. Controlled Fusion 2, 51 (1975).

${ }^{11}$ J. A. King (private communication, 2003).

${ }^{12}$ M. H. Key, F. Amiranoff, C. Andersen et al. in Proceedings of the 3rd Inertial Fusion Science and Applications Conference, Monterey, CA, September 2003, edited by B. Hogan (Institute of Physics, New York, 2004).

${ }^{13}$ M. E. Glinsky, Phys. Plasmas 2, 2796 (1995).

${ }^{14}$ G. Malka and J. L. Miquel, Phys. Rev. Lett. 77, 75 (1996).

${ }^{15}$ T. P. Hughes, S. S. Yu, and R. E. Clark, Phys. Rev. ST Accel. Beams 2 , 110401 (1999); D. R. Welch, D. V. Rose, B. V. Oliver, and R. E. Clark, Nucl. Instrum. Methods Phys. Res. A 464, 134 (2001).

${ }^{16}$ R. G. Evans (private communication, 2003).

${ }^{17}$ R. Kodama, K. Mima, K. A. Tanaka, Y. Kitagawa, H. Fujita, K. Takahashi, A. Sunahara, K. Fujita, H. Habara, T. Jitsuno, Y. Sentoku, T. Matsushita, T. Miyakoshi, N. Miyanaga, T. Norimatsu, H. Setoguchi, T. Sonomoto, M. Tanpo, Y. Toyama, and T. Yamanaka, Phys. Plasmas 8, 2268 (2001).

${ }^{18}$ Y. Sentoku, K. Mima, P. Kaw, and K. Nishikawa, Phys. Rev. Lett. 90, 155001 (2003).

${ }^{19}$ R. Edwards, in Proceedings of the 3rd Inertial Fusion Science and Applications Conference, Monterey, CA, September 2003, edited by B. Hogan (Institute of Physics, New York, 2004). 